2 Bergen Centre for Ethics and Priority Setting, Department of Global Public Health and Primary Care, University of Bergen, Bergen, Norway

3 Department of Medical Ethics and Health Policy, Perelman School of Medicine, University of Pennsylvania, Philadelphia

1Faculty of Philosophy, Oude Boteringestraat 52, University of Groningen, 9712 GL Groningen, Netherlands

Correspondence to: L Herzog l.m.herzog@rug.nl Cite this as: BMJ 2021;372:m4853 http://dx.doi.org/10.1136/bmj.m4853 Published: 05 January 2021

\title{
Covax must go beyond proportional allocation of covid vaccines to ensure fair and equitable access
}

\author{
International collaboration is key for the fair and efficient distribution of covid-19 vaccines. Lisa \\ Herzog and colleagues' Fair Priority Model, with its focus on allocating vaccine based on limiting \\ covid-19 harms, realises ethical principles better than Covax's proposal of proportional allocation \\ based on population
}

\section{Lisa M Herzog, Ole F Norheim, ${ }^{2}$ Ezekiel J Emanuel, ${ }^{3}$ Matthew S McCoy ${ }^{3}$}

The Covax Facility (Covax) is a multilateral initiative aimed at ensuring that all countries have "fair and equitable access" to covid-19 vaccines. Co-led by Gavi, the Vaccine Alliance (Gavi), the Coalition for Epidemic Preparedness Innovations (CEPI), and the World Health Organization (WHO), Covax is a voluntary arrangement that enables countries to pool their resources and risk by collectively investing in vaccine candidates while developing the political and logistical infrastructure needed for vaccine distribution. Most importantly, Covax ensures that vaccines financed through the initiative will be allocated in a transparent and coordinated manner.

In September 2020, WHO delineated its plan for allocating vaccines through Covax. ${ }^{1}$ Under the plan, vaccine doses would initially be allocated to participating countries in proportion to their population size. Only after each country receives vaccine doses for $20 \%$ of its population would countries' covid risk profiles be considered in a subsequent phase of vaccine distribution. Countries participating in Covax are permitted to pursue bilateral contracts with vaccine manufacturers like the one between the UK and Pfizer-BioNTech. Many countries, high income and even low-middle income countries, such as Indonesia and Vietnam, have secured vaccine through bilateral agreements.

Proportional allocation of vaccines through Covax is fairer and more efficient than an uncoordinated approach in which countries compete in the market to secure as much vaccine as possible for their own citizens. But proportional allocation fails to meet WHO's own ethical principles for vaccine allocation. An alternative approach to vaccine allocation, which we call the Fair Priority Model, would be better aligned with WHO's stated values and better suited to realising Covax's promise of fair and equitable access to covid-19 vaccines.

\section{Overcoming vaccine nationalism}

The commitment to international cooperation embodied in Covax is critical to preventing a future dominated by "vaccine nationalism," in which rich countries bid against each other to secure bilateral contracts with vaccine manufacturers and stockpile vaccine doses for their own citizens. ${ }^{2}$ Such vaccine hoarding by high income countries would be unfair because it would profoundly disadvantage people living in low income countries that lack the resources to procure vaccines for their citizens. It would also be inefficient because it would fail to allocate vaccines to countries with the greatest need and greatest potential for harm reduction, unnecessarily prolonging the global pandemic and causing many preventable deaths.

Compared with vaccine nationalism, the WHO plan for proportional allocation would be a major improvement in fairness and efficiency. First, it would ensure that each of the more than 170 countries participating in Covax would receive some vaccine in the initial phase of distribution-first for $3 \%$ of their population, with priority to health personnel, then for $20 \%$ of their population, with priority to high risk groups-regardless of their ability to pay. It would thus prevent a deeply unethical scenario in which large stores of vaccine are retained by high income countries while poorer countries, including those hit hardest by the pandemic such as Peru, have no or limited access to vaccines.

Second, modelling suggests that proportional allocation would be more efficient than an unregulated market and likely to reduce preventable deaths due to covid-19. Modellers recently compared a scenario in which the first two billion doses of covid-19 vaccine (assumed to be $80 \%$ effective two weeks after administration) are hoarded by the 50 wealthiest countries to one in which vaccines are distributed to all countries proportional to their population. ${ }^{3}$ They concluded that proportional allocation would avert nearly twice as many preventable deaths as hoarding by rich countries. Although there are substantial uncertainties about the effectiveness in real life circumstances of the vaccines that will eventually be produced-whether they will reduce the risk of transmission or death, in which groups, and how long immunity will last-these results support the comparative efficiency of the Covax model.

\section{Limitations of proportional allocation}

Despite its fairness and efficiency advantages over vaccine hoarding, proportional allocation falls short of key ethical principles in the WHO's Strategic Advisory Group of Experts' (SAGE's) own ethical framework for covid-19 vaccine allocation. ${ }^{4}$ Covid vaccines ought to be distributed according to their most likely function - to reduce death and serious economic and social harms. Distributing vaccine in proportion to a country's population might be a 
plausible way to implement equal concern in other contexts, but it is unjust for covid-19.

The SAGE framework incorporates six fundamental values: human wellbeing, equal respect, global equity, national equity, reciprocity, and legitimacy. Proportional allocation is poorly aligned with many, particularly the most substantive, of these ethical principles.

First, human wellbeing aims to "reduce deaths and disease burden" and "societal and economic disruption" due to covid-19. ${ }^{4}$ WHO's proportional allocation would require sending substantial quantities of vaccine to countries that have relatively low risk of death, disease, and social and economic disruption due to covid-19, such as New Zealand, Kenya, Senegal, South Korea, and Thailand instead of prioritising countries most affected by the pandemic, where the potential for harm reduction from a vaccine would be far greater, such as Mexico, Brazil, Iran, and Ecuador.

Second, equal respect aims at treating "the interests of all individuals and groups with equal consideration." 4 Treating people identically regardless of their circumstances is not equal respect. Rather, this principle requires responding to people's different needs with equal consideration. Those living in countries hardest hit by the pandemic have greater need for vaccines, in terms of both health and the economy, than those living in less severely affected countries. Proportional allocation fails to account for these differences in need and thus fails to treat people with equal respect.

Finally, global equity requires ensuring "that vaccine allocation takes into account the special epidemic risks and needs of all countries, particularly low and middle income countries."4 Ironically, proportional allocation disregards countries' special risks and needs. It pays no particular attention to the situation of individuals in low and middle income countries, who may have greater need for a vaccine than those living in other countries that are able to mitigate the most serious risks of the pandemic through effective public health measures.

\section{The Fair Priority Model}

As an alternative to WHO's proportional allocation scheme, we developed the Fair Priority Model (box 1). This model is better aligned with the SAGE group's fundamental ethical principles of human wellbeing, equal respect, and global equity.

\section{Box 1: The Fair Priority Model}

Based on three widely shared ethical values-benefitting people and limiting harm, prioritising those who are disadvantaged, and equal moral concern-the Fair Priority Model allocates vaccines between countries in three phases. Phase 1 aims at minimising premature deaths, based on the reduction of standard expected years of life lost (SEYLL) averted per dose. Phase 2 adds socioeconomic factors, measured in SEYLL, loss of gross national income (GNI), and reduction of the poverty gap. Phase 3 aims at returning countries to their pre-covid-19 situation.

Vaccine efficacy was not known when the model was proposed, so we assumed that approved vaccines would be safe and could reduce risk of severe complications and death from covid-19.

The Fair Priority Model has three stages of vaccine distribution. The aim of the first stage, assuming that vaccines will reduce mortality risk, is to reduce premature deaths caused directly or indirectly by covid-19 using standard expected years of life lost (SEYLL) averted per dose as a metric. SEYLL is commonly used in global burden of disease estimates. It calculates life years lost compared with a standardised global reference life table. Allocating vaccine on the basis of SYELL averted promotes the principle of human wellbeing by directing vaccine to the countries where it will save the most life years. It promotes the principle of equal respect by valuing a life saved at a given age identically, regardless of the country in which it occurs. Finally, because SEYLL's are standardised to the world's highest life expectancy, the lower life expectancy in many poorer countries is not taken as a reason to deprioritise those countries, which would violate the principle of global equity. Instead, by saving more life years, using SEYLLs would prioritise those living in the most disadvantaged countries.

In the second phase of distribution, the model takes serious economic and social harm caused by covid-19 into account by adding two socioeconomic metrics to SEYLL: improvements in gross national income (GNI) per dose and reduction in absolute poverty gap per dose. These indicators ensure that even after covid related deaths are brought relatively under control, vaccine will continue to be allocated in a way that prevents serious economic and social harms due to the pandemic while prioritising disadvantaged people by focusing on narrowing the poverty gap. Earlier vaccination can, for example, allow a poor country to relax lockdown measures that often hit the poorest part of the population who work in service sectors hardest.

In the third phase of distribution, the model aims to end community spread of covid-19 by prioritising countries with the highest transmission rates, ensuring that even as the pandemic begins to abate, priority is given to preventing harm and to people in countries most affected by the pandemic.

Thus, at each phase, the model gives priority to reducing the most harm to health and the economy with extra priority to those in the most disadvantaged countries. Moreover, it does so using well established metrics that can be transparently and consistently applied on the basis of available data.

\section{Pragmatic considerations}

Some people might argue that, despite its ethical limitations, proportional allocation is defensible on important pragmatic grounds. First, it might be claimed that the only way to assure the global community that the Covax mechanism will not arbitrarily favour some countries over others is to use a simple metric such as distribution by population size.

Any vaccine allocation plan must be based on clear metrics that can be consistently and transparently applied. But a mechanistic distribution of vaccine by population size is not the only way to satisfy this standard. The Fair Priority Model relies on metrics-SEYLL, GNI, and poverty gap-that are widely understood and already extensively used in global health. ${ }^{5}$ Additionally, under the current WHO proposal, the second phase of vaccine distribution is intended to take countries' covid risk profiles into account. Thus, the WHO proposal will eventually require metrics assessing the health and economic effects of covid, which will entail needing to use similar projections as the Fair Priority Model.

Second, some people claim that a major advantage of proportional allocation is that it gives high income countries an incentive to participate in Covax. Ensuring that all countries will have guaranteed access to a share of vaccines when they become available, might increase the likelihood that rich countries will agree to purchase vaccine through Covax. Acknowledging concern about wealthy countries' willingness to participate in Covax without such an incentive, WHO's chief scientist said that there was "a big, big risk that if you propose a very idealistic model, you may be left with nothing." 6

Covax leaders must be sensitive to political realities. Nevertheless, this pragmatic defence of proportional allocation seems flawed. 
Guaranteed access to doses for $3 \%$ or even $20 \%$ of their populations in an initial phase of vaccine allocation is unlikely to be a major incentive for high income countries. These levels of vaccination are too low to achieve herd immunity, not helping them escape the pandemic. More importantly, high income countries, such as Canada, Germany, Switzerland, and the United Kingdon, can secure doses for a much larger portion of their populations through direct, bilateral deals with drug companies-and in many cases they already have. In addition, even if high income countries do participate in Covax out of a sense of "enlightened self-interest,"7 they would have reason to favour an allocation scheme like the Fair Priority Model that would prioritise them if they experience an outbreak and have an urgent need for vaccine, rather than one that provides them with a fixed quantity of vaccine regardless of their level of need. Leaders of Canada, Germany, Switzerland, and the UK might reasonably ask why hundreds of thousands of very scarce vaccines held by Covax should go to Taiwan, which has had seven deaths, or New Zealand with 25 deaths, rather than their own countries with thousands of deaths.

\section{Conclusions}

Covax is a useful example of international cooperation. It will distribute vaccine more fairly and efficiently than vaccine nationalism. But Covax's proportional allocation fails to realise a fair and equitable distribution, and it cannot be defended on pragmatic grounds. To ensure fair and equitable access to covid-19 vaccines, Covax should consider adopting the Fair Priority Model. A renegotiation of Covax's terms of disbursement seems politically feasible expecially be cause countries know more about the global spread of covid and the needs of each country. This would require Covax to work closely with public health experts and modellers to decide where vaccines should be sent when they are manufactured, but this is entirely feasible and indeed necessary given the rapidily evolving dynamics of the pandemic.

\section{Key messages}

- Two main methods for allocatingCovid-19 vaccine among countries have so far been discussed: vaccine nationalism and WHO's proportional allocation scheme

- WHO's scheme is fairer and more efficient than vaccine nationalism, but fails to fulfill WHO's ethical principles of human wellbeing, equal respect, and global equity

- We suggest a third option, the Fair Priority Model, which distributes vaccine based on risk of premature death and economic disruption, and better realises WHO's ethical principles

Contributors and sources: This article was written by members of the FAAR project that developed the Fair Priority Model. LH is an economist and political philosopher with a focus on economic and social justice; OFN is a physician and medical ethicist with a focus on global health; EE is an oncologist and bioethicists with a focus on health policy and health justice; and MM is a political theorist and bioethicist with a focus on health policy. OFN and EE have extensive experience in giving policy advice on public health issues to governments and international organisations. This article draws on literature on global and health justice, the ethics of allocating scarce resources, and medical ethics. EE is the guarantor of the article.

Competing interests: We have read and understood BMJ policy on declaration of interests.

This work was partially funded by Wendy and Eric Schmidt and the Colton Foundation, which had no input into the content, writing, or review of the article. We thank other members of the FAAR project, especially Govind Persad, for helpful feedback.

1 World Health Organization.Fair allocation mechanism for covid-19 vaccines through the Covax Facility. 2020. https://www.who.int/publications/m/item/fair-allocation-mechanism-for-covid-19vaccines-through-the-covax-facility

2 Kupferschmidt K. 'Vaccine nationalism' threatens global plan to distribute COVID-19 shots fairly. Science. Published online July 28, 2020. Accessed October 4, 2020. https://www.sciencemag.org/news/2020/07/vaccine-nationalism-threatens-global-plan-distribute-covid-19shots-fairly
3 Gates B, Gates M. Vaccine fairness will make us all safer. Financial Times 2020 Sep 15 https://www.ft.com/content/f999c4e4-78a2-4f83-9beb-91c15dccd0b8

4 World Health Organization.WHO SAGE values framework for the allocation and prioritization of covid-19 vaccination. 2020. https://apps.who.int/iris/handle/10665/334299

5 Emanuel EJ, Persad G, Kern A, etal. An ethical framework for global vaccine allocation. Science 2020;369:1309-12. doi: 10.1126/science.abe2803. pmid: 32883884

6 Samuel S. Who should get the Covid-19 vaccine first? The equality vs. equity debate, explained Vox 2020 Sep 23. https://www.vox.com/future-perfect/21439799/covid-19-vaccine-priorityaccess-covax-who

$7 \quad$ World Health Organization. 172 countries and multiple candidate vaccines engaged in covid-19 vaccine global access facility. 24 Aug 2020. https://www.who.int/news/item/24-08-2020-172 countries-and-multiple-candidate-vaccines-engaged-in-covid-19-vaccine-global-access-facility 